\title{
REFERRALS TO PHYSIOTHERAPY: THE RELATION BETWEEN THE NUMBER OF REFERRALS, THE INDICATION FOR REFERRAL AND THE INCLINATION TO REFER
}

\author{
Jan J. Kerssens and Peter P. Groenewegen \\ Netherlands Institute of Primary Care, P.O. Box 1568, 3500 BN Utrecht, The Netherlands
}

\begin{abstract}
This article studies the relation between the referral rate and the type of patients general practitioners refer for physiotherapy. The study population consists of GPs participating in the Netherlands' Sentinel Stations Network, who recorded data on all referrals to physiotherapy during one year and filled in a questionnaire. Results show that the pattern of referral indications of high referring GPs does not differ systematically from that of low referring GPs. High referring GPs evaluate their patients complaints more as purely or mainly somatic. High referring GPs were no more inclined to give in to their patients demands, had busier practices, closer relations with physiotherapists and viewed their knowledge of physiotherapy as more satisfactory than low referring GPs. Some policy implications are discussed in respect to these results.
\end{abstract}

Key words-health professions, physiotherapy, general practice, referrals

\section{INTRODLCTION}

General practitioners vary considerably in their patterns of practice. Controlled for differences in the composition of their practice population or even for differences in the complaints presented by the patients, GPs differ in the interpretation of the complaints of their patients-e.g. according to the dimension somatic-psychosocial [1]-and in the kind of interventions, such as referrals or pharmaceutical prescriptions [2].

This general statement also applies to the number of referrals from GPs to physiotherapists and to the pattern of indications for referral [3]. The number of referrals to physiotherapists varies considerably between GPs. An analysis of the determinants of this variation has been reported in Kerssens et al. [4]. Not only does the number of referrals vary, but so does the pattern of indications for referral in terms of the diagnoses for which patients have been referred. An indication for this phenomenon is given in Kerssens et al. [5] who show the variation between GPs in the percentage of patients referred for purely somatic complaints, the percentage of referrals on demand of the patient, the percentage of referrals without a prescribed treatment and the variation for selected groups of diagnoses. These variations between general practitioners have not yet been studied systematically. In this article we present the first results of this study. Our analysis centres around the question whether the pattern of indications for referral differs for the group of GPs with the highest number of referrals, compared to the group with lowest number of referrals.

The study population consists of GPs participating in the Netherlands' Sentinel Stations Network [6]. These GPs have recorded data on all referrals to physiotherapists made during one year. We start this chapter with a short description of the position of
GPs and physiotherapists and the relations between them in the Dutch health care system (Section 2). In the next section the research question and hypotheses concerning the relation between referral rate and pattern of indications will be described (Section 3), while the fourth section is devoted to the methods of data collection, the classification of indications for referral and the methods of analysis. The fifth and the sixth sections contain the results of our analysis and a discussion of the result respectively.

\section{GENERAL PRACTICE AND PHYSICAL THERAPY IN THE DUTCH HEALTH CARE SYSTEM}

\section{General practitioners}

GPs have a central position in the Dutch health care system. Normally, they are the first professional health worker to contact in case of health problems and health related problems. A number of other health services, including physical therapy and medical specialists, are only accessable after a referral by a GP. In these respects the position of the Dutch GP resembles the situation in the United Kingdom or Denmark and differs from the situation in countries with direct access to medical specialists, such as Belgium, France or the U.S.A. Most of the GPs are in independent practice. Only $4 \%$ are in salaried service. The majority work in single-handed practice, but this majority is becoming smaller. The percentage of single handed practices decreased from $86 \%$ in 1970 to $55 \%$ in 1988 [7]. The remuneration of GPs is partly based on an annual capitation fee, for $65 \%$ of the population who are publicly insured, and partly on fee for service, for the privately insured part of the population [8]. Publicly insured patients have free health care, except for a small co-payment for pharmaceutical prescriptions and recently also for a refer- 
ral to a medical specialist. Privately insured patients pay their GP and depending on their private insurance policy may receive reimbursement. The number of GPs totalled 6275 in 1988. The list size averaged 2345 people.

\section{Physiotherapists}

The number of physiotherapists in the Netherlands is relatively high. The total number of active physiotherapists was estimated at 13,350 in 1986 [9]. Of the professionally active physiotherapists approximately two thirds work in primary care, numbering 8986 in 1987 [7]; the other third work in hospital or institutional settings.

The physiotherapists in primary care receive their patients through referrals, mainly from GPs $(80 \%$ of their patients), and in smaller numbers from specialists $(20 \%)$. The referred patient is introduced by a referral letter which constitutes the first contact between GP and physiotherapist. A quarter of the GPs do not restrict this contact to a referral letter; they also contact the physiotherapist before referral or in the course of treatment, or they accompany the referred patient with background information [10].

In the referral letter a diagnosis is stated and it may or may not include a prescription for the kind of physical therapy to be applied. If a prescription is included, the physiotherapist is legally obliged to follow it, unless there are very strong contra-indications in which case the physiotherapist should contact the GP. When the patient's therapy is finished the physiotherapist reports the results to the GP. For the legal position of physiotherapists in the Netherlands see Kortenhoeven [11]. The shared responsibility for the patient-the GP's for the diagnosis and the physiotherapist's for the treatment-is one of the principal characteristics of the relationship between the two professionals. In this respect their relationship differs from that between a GP and a medical specialist. Once a patient is referred to the medical specialist, the GP is, in most cases, no longer involved. Once a patient is referred for physical therapy this clearly should not be the case.

Before starting the therapy of a publicly insured patient, an authorization from the insurance company is necessary. Generally, these authorizations are granted for 12 combined treatment sessions (a combined treatment session consists of massage and/or exercise therapy and the application of a physical agent). Physiotherapists in primary care are remunerated on a fee for service basis. In the case of a privately insured patient, fees are directly charged to the patient who then, just as in the case of visits to a GP, depending on their insurance policy, can claim reimbursement. For the treatment of publicly insured patients, physiotherapists declare their services directly to the insurance company. The fee structure is based on the amount of time the different kinds of services are supposed to take; more time consuming services are costed higher $[12,13]$.

\section{RESEARCH QUESTION AND HYPOTHESES}

In an analysis based on data from the sentinel practices, the number of referrals based on the weekly recording of referrals and the actual composition by age and sex of the practice population and of referred patients were available. The age and sex composition is the most important determinant of the variation in the number of referrals. If the age and sex composition is taken into account, the variation is reduced to $60 \%$ of the original variation [14]. Still, there remain considerable differences between practices in the number of referrals to physiotherapists. Removing the influence of age and sex is a shortcut for controlling for situational differences between practices in health status of the practice population. The remaining variation is supposedly related to the decision process of the GP in interactions with patients and physiotherapists to whom he refers, and under the structural and situational conditions of practice.

To explore the remaining variation we pose the following question:

Is there a difference in the pattern of referral indications between GPs who have a relatively high referral rate and those with a relatively low referral rate, when the influence of the age and sex composition of the practice is controlled for?

There are two possible answers to this question and in both cases we are interested in hypotheses to explain the result.

If it turns out that GPs with a high number of referrals have a different pattern of indications compared to the GPs with a low number of referrals, our hypothesis is that the high referring GPs sce a broader range of conditions of patients as indications for referral to physical therapy. The decision to refer the patient is more often a relevant alternative for these GPs. The pattern of indications should differ in the sense that more patients are referred for conditions that are less commonly accepted as indications for physiotherapy and therefore fall out of scope as an alternative for others GPs.

When no difference in the pattern of referral indications between the high referring and the low referring group is found, it implies that high referring GPs refer more patients in all categories of the referral indications. The hypotheses to explain this result refer to the decision making situation (patient pressure, co-operative relations with physiotherapists, pressure of a busy practice) and to the perception and knowledge of GPs (evaluation of patients' complaints, knowledge of physiotherapy). Our first hypothesis to explain this result (if it turns out to be true), is that GPs with a high referral rate are, more inclined than their low referring colleagues, to give in to their patients demands to be referred to physical therapy. Our second hypothesis which does not exclude the first, is that the high referring GPs are possibly generally more inclined to refer patients for physical therapy, where other GP's for the same conditions of the patients would wait and see or first try some other therapy (rest, a diet, exercise, a prescription). The question then of course may be raised as to why these GPs are more inclined to refer. We will explore five possible explanations, based on: a different judgement of patients' complaints, a clinical approach rather than a general medicine approach, the pressure of a busy practice, relations with physiotherapists and knowledge of physical therapy. 
First of all, the GP's evaluation of the complaints of the patients as having a somatic or also a psychosocial background might influence his inclination to refer patients for physiotherapy. Evidence on the influence of these kinds of judgement on GPs' decisions is provided by Verhaak [15]. The hypothesis is that high referring GPs judge complaints of their patients more often as somatic problems and therefore belonging to the field of competence of physiotherapists. Our data, however, only pertain to referred patients. But research indicates that the evaluation of complaints in terms of somatic or psychosocial problems is a (rather) stable characteristic of the GP. A bias towards judging complaints as somatic problems is reflected by a clinical approach. Groll [16] developed a scale that measures the attitude of GPs to share responsibility with patients, revealing a general medicine or, in contrast, a clinical approach. We hypothesize that high referring GPs will show a clinical approach compared to low referring GPs who will show a more general medicine approach.

As far as the pressure of a busy practice is concerned, we hypothesize that the relation between the number of patients on the GP's list and the number of patients referred to physiotherapy is positive and conditional upon the number of patients on the list. The fourth and fifth explanation relates to the fact that high referring GPs are more knowledgeable about physical therapy and that GPs who know more about physical therapy are more inclined to refer patients. This could either be caused by closer co-operation with physiotherapists or by a generally higher level of knowledge about the possibilities of physical therapy. There is evidence that knowledgeable physicians refer more patients and that they tend to be prescriptive, directing the physiotherapist as to what treatment to apply [17]. However, if high referring GPs co-operate more closely with physiotherapists, this might also result in a less directive style of referral due to a relation of mutual trust.

We summarize the preceding discussion in Fig. 1.

\section{METHODS}

During 1985, participants in the Sentinel Stations Network recorded a number of data on each referral for physical therapy. The sex, age and insurance status of all referred patients were recorded on a weekly recording form. A distinction was made between new referrals and referrals for continuation of current treatments. This distinction relates to regulations of the public health insurance system, requiring a new referral and authorization, usually after 12 combined treatment sessions [13]. New referrals are defined as being the first time a patient is referred or referrals after a period free of complaints. Additional data on each new referral has been recorded:

-the GP's diagnosis of the patient's complaint (later on coded in the ICD-9-CM) [18];

-source of the initiative for the referral (a 5-point scale ranging from the GP's initiative to intiative of the patient);

-the extent to which psychosocial aspects are involved (a 6-point scale developed by Verhaak) [12];

- whether or not a treatment was prescribed and, if so, what treatment.

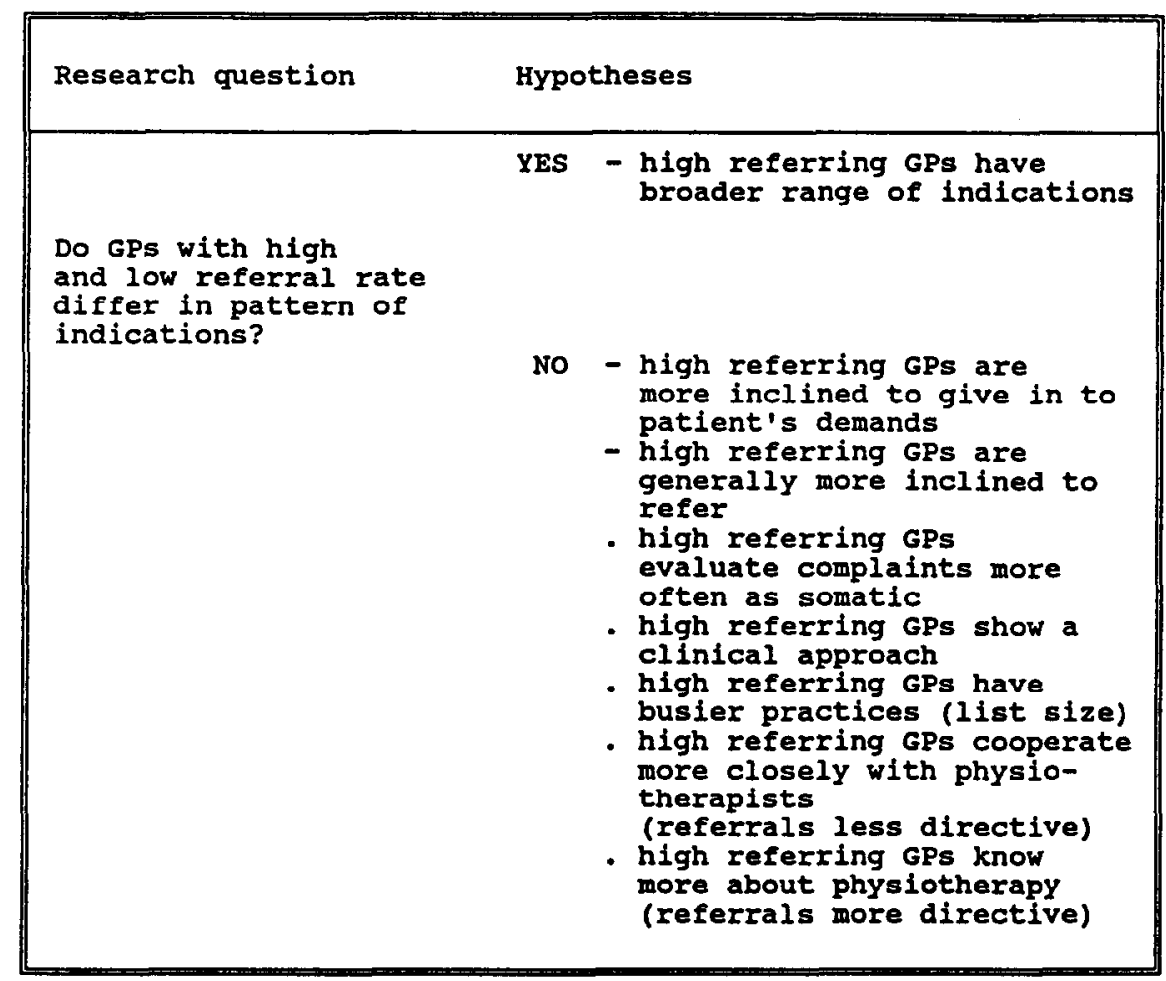

Fig. 1. Summary of research questions and hypotheses. 
The GPs also respond to a mailed questionnaire. The following topics are of interest:

- the range of indications GPs regard as suitable for referral for physical therapy, measured by their response to 20 short case descriptions;

-closeness of co-operation with physiotherapists;

-the GP's own evaluation of his knowledge of physical therapy;

- the clinical versus general medicine approach.

In short, there are three kinds of data: all recorded referrals, additional data on new referrals, and the questionnaire. Data on all recorded referrals were only used to standardize the referral rates. Since the composition of the practice population in terms of age and sex is known, we are able to standardize referral rates and thus establish whether or not a GP refers more or less than is to be expected according to the age-sex distribution of his patients [19].

To answer the question concerning the pattern of indications, we aggregated the number of new referrals with a specific diagnosis and made a percentage distribution of the 25 most common diagnoses for each GP. GPs with a relatively low number of new referrals exhibit many zeros in this distribution which impedes our analysis to a large extent. We solved this problem by forming three groups of GPs. In the 'low' group we categorized general practitioners (totalling 14) who referred less patients than would be expected from their practice population. GP's who referred about the same patients as expected (totalling 15), fell into the category 'medium'. The 'high' group consists of GPs (totalling 16) who referred more patients than expected.

Diagnoses were classified in the International Classification of Disease and Causes of Death, the ninth revision, Clinical Modification. The ICD-9-CM was selected because it differentiates among more disorders and diseases than any other classification. From a physical therapy point of view, the ICD is not completely adequate [20]. The main criticism is related to the section on 'dorsopathies'. This inadequacy has been solved by classifying some disorders in the sections on 'arthropathies' and 'rheumatism' with additional digits to indicate the location (spine, sacroiliac joint and back).

\section{Statistical analysis}

In the following section results are presented mainly as percentages. Percentages have the advantage of being easy to interpret; the practical significance of differences is easily determined by the use of percentages. The reader may wonder if the differences are statistically significant. It is important to keep in mind that we have data on the level of new referrals and on the level of GPs. Statistical significance depends on the way in which we answer the research question and hypotheses. When we give answers in terms of more than 6000 new referrals, all the differences, even small and unsystematic ones, are statistically significant. See for example Bakan [21] for illustrations of this phenomenon. The main research question and the first three hypotheses are answered in terms of additional data on new referrals. For these hypotheses, statistical significance is an inadequate criterion.
When we give answers in terms of subgroups of GPs only very large differences (more than $40 \%$ ) are statistically significant. So in this case statistical insignificant differences still can be of considerable practical interest. The last four hypotheses are answered in terms of GPs. We do not therefore use significance in the statistical sense as the criterion for the acceptance or rejection of our specific hypotheses. We will however use the sign test to check whether the number of confirmed hypotheses, based on the GPS as units of observation, is higher than can be expected by chance.

\section{RESULTS}

Our GP's diagnoses relate to 6397 new referrals (diagnoses were missing for a small number). More than 600 diagnoses were differentiated in the ICD-9$\mathrm{CM}$ classification. Diseases or disorders of the musculoskeletal system are most frequent in more than $90 \%$ of the patients. To reduce the vast number of diagnoses, without simplifying it too much from a physiotherapist's point of view, we have used the 25 most common diagnoses (or small groups of diag. noses). Table 1 gives the percentage distribution of these diagnoses for high, medium and low referring GPs.

Inspection of the table shows the differences between groups of GPs to be very small and not systematic. The answer to the central question is therefore that there is no difference between high and low referring GPs in the pattern of referral indications [22]. Given this answer there is no reason to suppose a different range of referral indications be-

Table 1. Indication for referral in percentages for three types of GPs according to their standardized referral rates. Figures relate to percentages of new referrals

\begin{tabular}{|c|c|c|c|}
\hline \multirow[b]{2}{*}{ Disorders } & \multicolumn{3}{|c|}{ Referral rate } \\
\hline & $\begin{array}{l}\text { Low } \\
(\%)\end{array}$ & $\begin{array}{c}\text { Medium } \\
(\%)\end{array}$ & $\begin{array}{l}\text { High } \\
(\%)\end{array}$ \\
\hline Muscle/soft tissue (back) & 10 & 12 & 11 \\
\hline Muscle (low back) & 10 & 12 & 11 \\
\hline Enthesopathy (shoulder) & 8 & 7 & 6 \\
\hline Cervico-brachial syndrome & 5 & 3 & 4 \\
\hline Spondylosis & 3 & 5 & 2 \\
\hline Intervertebral disc & 5 & 3 & 2 \\
\hline Enthesopathy (elbow) & 3 & 3 & 3 \\
\hline Curvature of spine & 2 & 3 & 3 \\
\hline Pain in spine'sacro-iliac & 3 & 3 & 3 \\
\hline Sciatica & 2 & 2 & 3 \\
\hline Respiratory system & 4 & 2 & 2 \\
\hline Muscle/soft tissue (shoulder) & 1 & 3 & 2 \\
\hline Muscle/soft tissue (pelvic) & 2 & 2 & 2 \\
\hline Neuritis & 2 & 3 & 1 \\
\hline Osteoarthrosis knee & 3 & 2 & 2 \\
\hline Contracture spine & 1 & 2 & 2 \\
\hline Sprains and strains of ankle & 1 & $i$ & 2 \\
\hline Derangement knee & 1 & 2 & 2 \\
\hline Migraine/headache & $i$ & 2 & 2 \\
\hline Osteoarthrosis hip & $i$ & 1 & 1 \\
\hline Enthesopathy (knee) & $i$ & $i$ & 2 \\
\hline Enthesopathie (ankle) & 1 & 1 & 1 \\
\hline Enthesopathie (hip) & 1 & $i$ & 1 \\
\hline Hyperventilation & 1 & 1 & $i$ \\
\hline Muscle/soft tissues (neck) & 1 & 1 & $i$ \\
\hline Classified elsewhere & 28 & 26 & 30 \\
\hline Total & 100 & 100 & 100 \\
\hline$N$ (new referrals) & 1130 & 1756 & 3606 \\
\hline$N$ (practitioners) & 14 & 15 & 16 \\
\hline
\end{tabular}


tween high and low referring GPs. Therefore there is also no use in exploring the hypothesis that high referring GPs regard a broader range of indications as fit for referral to physiotherapy.

To account for the fact that there is no difference in referral pattern we have first of all hypothesized that high referring GPs are more inclined to give in to their patient's demands. To test this hypothesis we looked at the percentage of referrals that were made at the initiative of the patient rather than of the GP (scale values 4 and 5 ). This percentage is $40 \%$ for the low referring group, $37 \%$ for the middle group and $39 \%$ for the high referring GPs. This hypothesis cannot be accepted.

To find out whether high referring GPs have a different way of evaluating their patient's complaints, we looked at the percentage of referrals where the GP evaluated the complaints as purely somatic (scale value 1). For the low referring group of GPs this percentage was $48 \%$, for the middle group $51 \%$ and for the high referring group $60 \%$. The differences were as predicted and the middle group is indeed in between the two extremes. It should be noted that the hypothesis was more broadly formulated than the data used to test it; the hypothesis was that high referring GPs generally evaluate the complaints of all their patients (not only those who were referred) in more somatic terms. The hypothesis that high referring GPs exhibit a clinical approach in contrast to the low referring group was not rejected, although the difference in this respect was only small: $38 \%$ of the high referring group, $31 \%$ of the medium and $31 \%$ of the low referring GPs revealed a clinical approach. These results indicate that the evaluation of referred patients' complaints on the dimension somatic-psychosocial has more influence on the referral rate than the GP's approach.

The next hypothesis links list size to referral rate. List sizes have been classified into small, medium and large. The association of list size and referral rate proved to be slightly positive; $44 \%$ of the high referral group have large practices and $29 \%$ of the low referral group have large practices. The medium group was not quite consistent in this respect because only $13 \%$ had large practices. There is some support for this hypothesis [23].

The next hypothesis stated that high referring GPs co-operate more closely with physiotherapists. Cooperative relations between the GPs and physiotherapists in relation to the referral rates was tested in four different ways:

-the number of physical therapy practices patients are referred to;

-the priority of working with physiotherapists as compared to other primary care professionals (district nurse, social work);

-the extent to which discussion of referred patients cases takes place;

-the presence of a physiotherapist in the GP's social network.

We would posit that a GP with close co-operative relations with physiotherapists refers to a small number of physical therapy practices, gives high priority to working with physiotherapists and communicates with the physiotherapist when he refers a patient. Finally the presence of a physiotherapist in his social network covers their relationship from a non-professional point of view. High referring GPs should show more social relations with physiotherapists than their low referring colleagues.

Our data show that $62 \%$ of the GPs in the low referring group refer their patients to 3 or fewer practices, whereas $44 \%$ of the GPs in the high referring group refer their patients to 3 or fewer practices. This result contradicts our hypothesis that high referring GPs have closer relations. It might be suggested that high referring GPs, to meet the demand they create, need more than a few physiotherapists on call. But because the average physical therapy practice with 2 or 3 physiotherapists can easily handle the demand of 3 or 4 GPs, this suggestion seems very unlikely. As far as the priority of co-operating with physiotherapists and discussing patients cases is concerned, our assumptions are confirmed: $52 \%$ of the high referring GPs state that this priority is high in respect of $39 \%$ of the low referring GPs. $63 \%$ of the high referring GPs give the physiotherapists background information on the patients referred compared to $36 \%$ of the low referring GPs. When we look at the presence of physiotherapists in the GPs social network $72 \%$ of the GPs in the high referring group have a physiotherapist in their social network compared to $51 \%$ of the GPs in the low referring group.

Finally we look at the directiveness of referrals. According to our hypotheses high referring GPs were either more directive (where high referring GPs are also more knowledgeable about physiotherapy) or less directive (when high referring GPs work closer together with physiotherapists). The percentage of referrals without a prescribed treatment turned out to be $21 \%$ for the low referring GPs, $25 \%$ for the middle group and $51 \%$ for the high referring group. This means that our assumption about high referring GPs and the mechanics of mutual trust is not contradicted. High referring GPs have both a closer relation with physiotherapists and are less prescriptive in their behaviour towards physiotherapy. To see if knowledge of physiotherapy is an influence on its own, we present the last percentages. $72 \%$ of the high referring GPs consider their knowledge of physical therapy satisfactory and $47 \%$ of the low referring group make a similar assessment of their own knowledge. The medium group is not quite consistent in this respect, since $75 \%$ of the GPs regard their knowledge as satisfactory. We conclude that our assumption about knowledgeable GPs is confirmed. However, the hypothesis that the high referring GPs are in consequence more directive in their referrals, is not confirmed.

Table 2 summarizes the information on the hypotheses discussed in the foregoing text and gives an evaluation of the hypotheses. Of all hypotheses based on the GP as our unit of observation, only one is clearly rejected. The difference for the hypothesis on clinical approach is relatively small, but in the predicted direction. The probability of six out of seven hypotheses falling in the predicted direction by chance only is 0.06 . 
Table 2. Evaluation of the hypotheses. The three columns with percentages relate to three types of GPs practitioners according to their standardized referral rates

\begin{tabular}{|c|c|c|c|c|}
\hline & \multicolumn{3}{|c|}{ Referral rate } & \multirow[b]{2}{*}{$\begin{array}{l}\text { Hypothesis confirmed (C)/ } \\
\text { rejected(R) }\end{array}$} \\
\hline & $\begin{array}{l}\text { Low } \\
(\%)\end{array}$ & $\begin{array}{c}\text { Medium } \\
(\%)\end{array}$ & $\begin{array}{l}\text { High } \\
(\%)\end{array}$ & \\
\hline \multicolumn{5}{|c|}{$\begin{array}{l}\text { Hypotheses answered in terms of new referrals } \\
\text { Indication for referral }\end{array}$} \\
\hline (e.g. class, else)* & 28 & 26 & 30 & $\mathbf{R}$ \\
\hline Patient-initiative & 40 & 37 & 39 & $\mathbf{R}$ \\
\hline Purely somatic & 48 & 51 & 60 & C \\
\hline Non-directive referral & 21 & 25 & 51 & $\mathrm{C} / \mathrm{R}+$ \\
\hline$N$ (new referrals) & 1130 & 1756 & 3606 & \\
\hline \multicolumn{5}{|c|}{ Hypotheses answered in terms of GPs } \\
\hline Clinical approach & 31 & 31 & 38 & C \\
\hline Large practice & 29 & 13 & 44 & C \\
\hline Refers to $<3$ practices & 62 & 43 & 44 & $\mathbf{R}$ \\
\hline Priority high & 39 & 43 & 52 & $\mathrm{C}$ \\
\hline Communicates & 36 & 64 & 63 & C \\
\hline Friend & 51 & 54 & 72 & C \\
\hline Knowledge satisfactorily & 47 & 75 & 72 & C \\
\hline$N$ (practitioners) & 14 & 15 & 16 & \\
\hline
\end{tabular}

\section{DISCUSSION}

With regard to our main research question, we conclude that high referring GPs do not refer qualitatively different categories of patients (in terms of disorders) than their low referring colleagues. To explore in which way high referring GPs differ from low referring GPs, we found that high referring GPs are no more inclined to give in to their patients demands. High referring GPs evaluate their patients complaints more as purely or mainly somatic and are slightly more clinical in their approach. Furthermore, the results point to the fact that high referring GPs have busier practices, have closer co-operative relationship with physiotherapists and evaluate their knowledge of physiotherapy as more satisfactory than low referring GPs.

In our analysis we did not bother much about statistical significance. Our main research question was answered in terms of subgroups of new referrals totalling up to more than 6000 . With such a large number even small and unsystematic differences are statistically significant. The reader can judge the practical significance of these differences by inspecting Tables 1 and 2. On the other hand some of our hypotheses were answered in terms of subgroups of GPs. With only 45 GPs none of the differences were statistically significant but some of them were, at least in our view, of practical significance. The small number of GPs made it also impossible to apply multivariate statistical analysis, so we were not able to determine the relative importance of our independent variables in a more comprehensive way. This will await further analysis in an ongoing project [24].

In general one can state that the variation in referral rates is generated by differences in the practice population and by differences in characteristics of GPs (leaving aside structural factors such as supply of physical care [25]). In this paper the emphasis has been on characteristics of GPs. We did not consider a principal factor like morbidity in the practice population as one of the explanatory factors for variation in referral rates. As the morbidity of the practice population is unknown, we used the age-sex distribution as a proxy. Given the fact that this proxy reduced the variation between GPs to a considerable extent, the question arises as to how much of the remaining variation could be explained when morbidity is checked. Furthermore our data showed that high referring GPs judged their patients complaints in more somatic terms than low referring GPs. These judgements were only slightly associated with a general bias towards expressing complaints in somatic terms (the clinical approach). An alternative explanation is that high referring GPs see more patients with musculoskeletal disorders and consequently refer more to physiotherapists than their colleagues, an explanation which cannot be checked.

In this section we will briefly consider two items. The fact that the presence of a physiotherapist in the GP's social network is associated with a relative high number of referred patients seems not to be very useful and even irrelevant [26]. But observations like this point to the limits on policy making. If variation between GPs in the number of referred patients is caused by factors like this, an attempt to influence this variation is beyond the scope of legislation or incentive programmes.

Ritchey et al. [3] conclude: “... greater professional autonomy is likely to be acquired by physical therapists making physicians aware of the extent of therapists' capabilities...". This conclusion was reached on the basis of the fact that knowledge of the concrete pragmatic services that physical therapists can provide is the decisive variable in increasing referrals at all levels of competency. Our data too, showed the knowledgeable GP as a relatively high referrer. Most GPs judge their knowledge after graduating from university as unsatisfactory [4]. Their knowledge improves with their experience and they regard the physiotherapists as their main source of information. This explains why knowledge and 
close-co-operation co-vary with one other. Of course the validity of a GP's own judgement on his knowledge of physical therapy can be questioned. Stanton et al. [27] show however a deficit in resident physician's knowledge of physiotherapy in a more valid way. If we assume that GPs can accurately judge their knowledge in this respect, the policy of improving this knowledge seems worthwhile.

There are several ways in which knowledge of physical therapy options can be transferred to GPs. Of course a better training in physical therapy is one option. Uili et al. [17], however, state that physicians with more knowledge of physical therapy procedures are those with more years in the field, so intervention in the medical school is less reasonable.

Another option is to carry out clinical trials in physical therapy to ascertain the effects of physiotherapy for different kinds of patient. The outcomes of such studies should then be communicated to GPs to enable the provision of care in a standardized form.

Yet another option is to create conditions in which the GP and the physiotherapist can come to a better understanding of each other. Lack of understanding is reported in Bourne [28] in an evaluation of a physiotherapy seminar where difficulties with doctors rather than patients were conspicuous.

In the present situation the physiotherapists main emphasis is on the treatment of patients. In most cases this treatment will take approx. 4-8 weeks. When the treatment is finished the physiotherapist sends a report to the GP. Feedback about the referred patient will only come to the attention of the GP much later. This feedback-loop can be shortened by sharing the responsibilities for the indication for physical therapy more equally between GP and physiotherapist. In this option a patient is not referred until both GP and physiotherapist examine the patient and consult one other about the best treatment for the patient. This strategy should not be followed automatically, it should only be applied in cases where the GP is insecure as to whether or not physical therapy can improve the patients' health status. If physical therapy can improve the patients health status (according to both GP and physiotherapy) under-utilization of physical therapy is reduced. But if both GP and physiotherapist conclude that there is little chance that physical therapy will improve the patient's health status they should refrain from treatment to reduce over-utilization. This means a reconsideration of the process of delivering physical therapy care in the sense of extending the options of referral for consultation purposes only. In this process the number of prescriptive referrals probably decreases, which might enhance the professional status and authority of physiotherapists.

\section{REFERENCES}

1. Verhaak P. F. M. Interpretatie en behandeling van psychosociale klachten in de huisartsenpraktijk: een onderzoek naar verschillen tussen huisartsen. Nivel, Utrecht, 1985.

2. Wilkin D. and Smith A. Explaining variation in general practitioner referrals to hospital. Fam. Pract. 4, 160-169, 1987.

3. Ritchey F. J., Pinkston D., Goldbaum J. E. and Heerten M. E. Perceptual correlates of physician referral to physical therapists: implication for role expansion. Soc. Sci. Med. 28, 69-80, 1989.

4. Kerssens J. J., Groenewegen P. P. and Curfs E. Chr Determinanten van het aantal verwijzingen van huisartsen naar fysiotherapeuten. Tijdschr. Soc. Gezondh. 65, 455-461, 1987.

5. Kerssens J. J., Curfs E. Chr. and Groenewegen P. P. Fysiotherapie in de Nederlandse Gezondheidszorg. klachten van patienten, indicatiestelling van (huis) - art. sen en fysiotherapeutische behandelingen. Nivel, Utrecht, 1987.

6. Continuous Morbidity Registration, Sentinel Stations. The Netherlands, 1985. Utrecht, Nivel.

7. Dam F. van and Hingstman L. Cijfers uit de registratie van beroepen in de eerstelijnsgezondheidszorg 1988. Nivel, Utrecht, 1988.

8. Rutten F. and Werff A. van der. Health Policy in the Netherlands: at the crossroads. In The Public/Private Mix for Health: the Relevance and Effects of Change (Edited by McLachlan G. and Maynard A.). The Nuffield Provincial Hospitals Trust, London, 1987.

9. Ministerie van Welzijn, Volksgezondheid en Cultuur. Notitie: Taakstelling Beroepskrachten planning Gezondheidszorg 1988. WVC, Rijswijk, 1988.

10. Groenewegen P. P., Kerssens J. J. and Curfs E. Chr. Fysiotherapie in de Nederlandse Gezondheidszorg. Nederlands Tijdschr. Fvsiother. 99, 22-32, 1989.

11. Kortenhoeven D. Verboden toegang voor onbevoegden: een studie over de voorstellen tot verruiming van het wettelijk kader van de medische en paramedische beroepsuitoefening. N.H.I., Utrecht, 1982.

12. Curfs E. Chr. and Groenewegen P. P. Physiotherapy in the Netherlands: an overview. Physiother. Pract. 2, 132-137, 1986.

13. Doorslaer E. van and Geurts J. Supplier-induced transformation of demand evidence on dutch physiotherapists. Soc. Sci. Med. 11, 919-925, 1987.

14. Kerssens J. J. and Curfs. E. Chr. Fysiotherapie in de Nederlandse Gezondheidszorg. Nivel, Utrecht. In preparation.

15. Verhaak P. F. M. Variations in the diagnosis of psychological disorders: a general practice observation study. Soc. Sci. Med. 23, 595-604, 1986.

16. This is a Likert-type scale which contains nine items, originally developed by: Grol R. Preventie van de somatische fixatie: en de attitude van de huisarts. N.U.H.I. Nijmegen, 1983. In our sample the reliability (Cronbach's alpha) of this scale is 0.70 , as shown by: Schrijnemaekers V. Het verwijscijfer van de peilstations nader onderzocht. Nivel, Utrecht, 1987 (technical report).

17. Uili R. M., Shepard K. F. and Savinar E. Physician knowledge and utilization of physical therapy procedures. Phys. Ther. 64, 1523-1530, 1984.

18. International Classification of Diseases-9th versionClinical Modification. Commission on Professional and Hospital Activities, Michigan, 1979.

19. The expected number of patients were calculated as follows. We formed proportions by dividing the number of referred patients (for all the GPs together) by the number of patients on all the practice population lists together for each sex-age category. With the assumption that GPs do not differ in their referral strategy, these proportions are the same for each GP. We then calculated the expected number of referrals by multiplying these proportions with the number of patients on each of the GPs' list for each sex-age category. Finally we computed the sum of these expected numbers for each GP. We then compared the expected number of referrals with the recorded number of referrals.

20. Curfs E. Chr., Groenewegen P. P. and Kerssens J. J. Fysiotherapie in de Nederlandse Gezondheidszorg: classificatie van gegevens in onderzoek op het gebied van de fysiotherapie. Nivel, Utrecht, 1987. 
21. Bakan D. The test of significance in psychological research. Psychol. Bull. 66, 423-437, 1966.

22. We have come to this conclusion in spite of the statistical significance of the differences. Due to the overwhelming power of our analysis even very small differences are significant.

23. Due to small power these differences are not statistically significant.

24. Foets M., Velden J. van der and Zee J. van der. A National Study of Morbidity and Interventions in General Practice. Nivel, Utrecht, 1986.

25. In an analysis of both structural factors and characteristics of GPs in relation the number of referrals to physiotherapists, the accessibility of physiotherapeutical care and the type of practice the GP works in showed a relation with referral rates $[4,10]$.

26. Wolff A. N. de. De waarde van de fysiotherapie in de nederlandse gezondheidszorg. In Gaarne Fysiotherapie... (Edited by Bol F. A., Melker R. A. de, Sneep R. and Wolff A. N. de). Samson Stafien, Alphen aan den Rijn, 1989

27. Stanton P. E., Fox F. L., Frangos K. M., Hoover D. H and Spilecki G. M. Assessement of residents physicians knowledge of physicaltherapy.Phys. Ther 65,27-30,1985.

28. Bourne S. Doctors, physiotherapists and placebo pharmacology. Br. med. J. 2, 1761-1763, 1978. 